\title{
Extractos de Sargassum spp. como inductores de tolerancia a Fusarium oxysporum en plántulas de tomate
}

\section{Sargassum spp. extracts as tolerance inducers to Fusarium oxysporum in tomato seedlings}

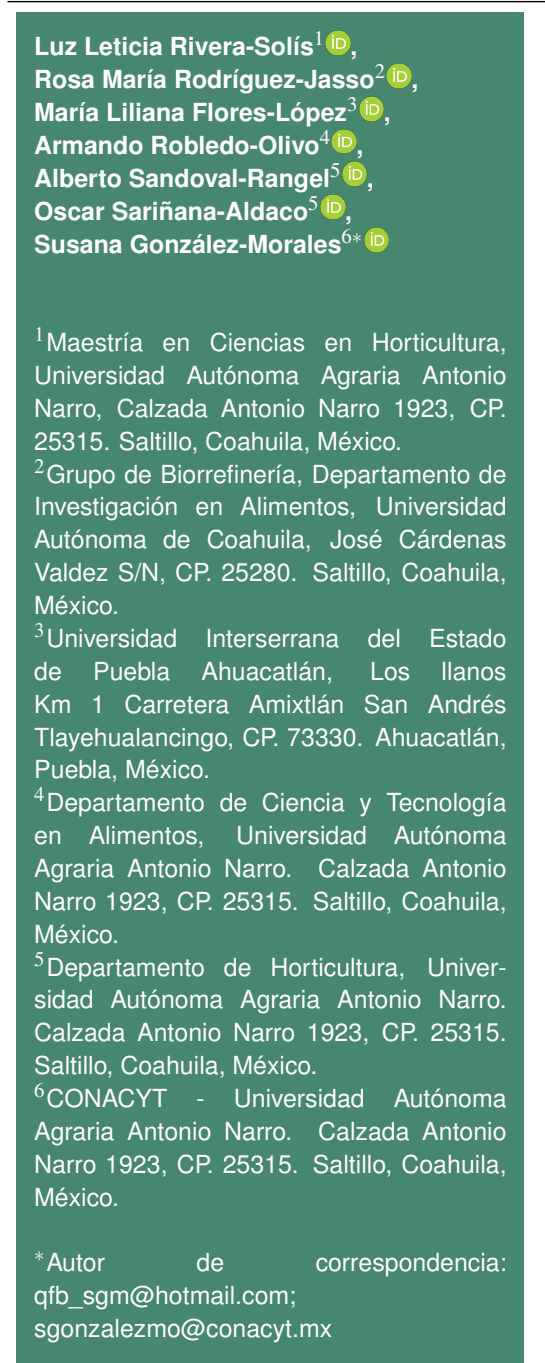

Nota científica

Recibida: 29 de enero 2021

Aceptada: 26 de abril 2021

Como citar: Rivera-Solís LL, RodríguezJasso RM, Flores-López ML, RobledoOlivo A, Sandoval-Rangel A, SariñanaAldaco O, González-Morales S (2021) Extractos de Sargassum spp. como inductores de tolerancia a Fusarium oxysporum en plántulas de tomate. Ecosistemas y Recursos Agropecuarios 8(1): e2826. DOI: 10.19136/era.a8n1.2826
RESUMEN. Fusarium oxysporum es uno de los patógenos más destructivos con gran distribución y efectos devastadores en hortalizas. Una alternativa para mitigar el daño por $F$. oxysporum es el uso de bioestimulantes, ya que promueven una respuesta de defensa en situaciones de estrés. El objetivo del presente trabajo fue evaluar el potencial bioestimulante de extractos hidroalcohólicos de Sargassum spp. (SAR) para incrementar la tolerancia a la infección por $F$. oxysporum en plántulas de tomate. Se evaluaron 17 extractos, los cuales se aplicaron de manera foliar a las plántulas a los 7, 12, 22, 33 y 43 DDT (días después del trasplante). La inducción de tolerancia de los extractos de SAR se evaluó en términos de incidencia y severidad de la enfermedad y variables agronómicas. El extracto E12 mostró un mejor control de la enfermedad con un $17 \%$ menos de la severidad de síntomas respecto al testigo infectado.

Palabras clave: Bioestimulante, extractos hidroalcohólicos, estrés biótico, Solanum lycopersicum, incidencia y severidad.

ABSTRACT. Fusarium oxysporum is one of the most destructive pathogens with wide distribution and devastating effects on vegetables. An alternative to mitigate the damage caused by $F$. oxysporum is the use of biostimulants, since they promote a defense response in stressful situations. The objective of this work was to evaluate the biostimulant potential of hydroalcoholic extracts of Sargassum spp. (SAR) to increase tolerance to infection by $F$. oxysporum in tomato seedlings. 17 extracts were evaluated, which were applied in a foliar way to the seedlings at 7, 12, 22, 33 and 43 DAT (days after transplantation). The induction of tolerance of the SAR extracts was evaluated in terms of incidence and severity of the disease and agronomic variables. The E12 extract showed better disease control with $17 \%$ less severity of symptoms with respect to the infected control.

Key words: Biostimulant, hydroalcoholic extracts, biotic stress, Solanum lycopersicum, incidence and severity. 


\section{INTRODUCCIÓN}

La agricultura enfrenta el desafío de proporcionar alimentos a una población mundial creciente, además de enfrentar superar numerosos estreses bióticos y condiciones ambientales adversas (González de Molina et al. 2017). El estrés biótico es causado por el ataque de una amplia gama de plagas y patógenos, entre los que destaca la presencia de hongos como las principales causas de pérdidas en los cultivos (Kumar et al. 2018). Las especies del género Fusarium están ampliamente dispersas en los suelos de todas las zonas climáticas a nivel mundial, y se asocian con una gran diversidad de plantas a las que causan graves enfermedades (Summerell 2019). Es uno de los patógenos más destructivos con gran distribución y efectos devastadores en hortalizas, causante de la marchitez vascular, reconocida como la principal enfermedad que origina problemas en el cultivo de tomate (Ammar et al. 2017).

El tomate es la segunda hortaliza más cultivada a nivel mundial, pero es susceptible durante el cultivo o posterior a la cosecha a más de 200 enfermedades causadas por hongos, bacterias, nemátodos y virus fitopatógenos (Singh et al. 2017). Durante décadas se ha visto un gran crecimiento en el uso de agroquímicos a nivel mundial, sin embargo, el uso indiscriminado de éstos, específicamente los plaguicidas, ha provocado la acumulación de residuos tóxicos en el suelo, aire, agua y alimentos, así como la generación de resistencias en plagas (Campos et al. 2019). Por lo anterior, se requiere ofrecer alternativas sostenibles al uso de agroquímicos convencionales hacia una industria agrícola más segura (Maluin y Hussein 2020).

En la búsqueda de alternativas para disminuir la severidad de las enfermedades causadas por patógenos (estrés biótico), se ha estudiado la inducción de tolerancia al estrés en las plantas con la aplicación de bioestimulantes (Ali et al. 2020). Dando como resultado la alteración de los procesos metabólicos que dan lugar al uso más eficiente de recursos ambientales, crecimiento o rendimiento sus- tancialmente mayor y un incremento en la tolerancia a factores ambientales adversos (Juárez-Maldonado et al. 2019). Recientemente se ha estudiado a las algas pardas para el desarrollo de bioestimulantes, debido a que representan una fuente potencial de compuestos bioactivos con una amplia variedad de actividades biológicas, que pueden ser eficaces para controlar las enfermedades de las plantas (Melo et al. 2020). Las algas y sus derivados también se utilizan en la agricultura como potenciales fertilizantes, bioestimulantes, mejoradores de suelo y pesticidas en las plantas (Dmytryk y Chojnack 2018). Como el género Sargassum, característico en la producción de metabolitos secundarios con alto potencial biotecnológico (Nawaim et al. 2017). En este tenor, Sargassum spp. representa una fuente potencial de explotación debido a su alta disponibilidad y contenido de compuestos bioactivos (Rodríguez-Martínez y van Tussenbroek 2016). Entre los componentes encontrados en las algas se incluyen: polisacáridos, vitaminas, minerales, proteínas, antioxidantes, pequeñas cantidades de lípidos, polifenoles y pigmentos (García-Enciso et al. 2018). En la caracterización de algunos de sus compuestos como los polisacáridos y oligosacáridos, particularmente el caso del ulvano, laminarinas, fucanos sulfatados, carragenanos y alginatos, se estudió la capacidad de desencadenar respuestas de defensa, consiguiendo suprimir enfermedades a través de la acción directa sobre los agentes causales e inducir tolerancia al estrés biótico y abiótico en plantas (Nawaim et al. 2017, Bouissil et al. 2020). Estudios previos muestran que los extractos de algas pueden activar o interactuar con los mecanismos de defensa latentes de las plantas, induciendo resistencia frente al estrés, lo que hace que la planta sea menos susceptible al ataque posterior de patógenos (Rehman et al. 2017, Melo et al. 2020). Por lo anterior, el objetivo del presente trabajo fue evaluar el potencial de extractos hidroalcohólicos de SAR en base al control de incidencia y severidad para incrementar la tolerancia a la infección por $F$. oxysporum en plántulas de tomate. 


\section{MATERIALES Y MÉTODOS}

\section{Producción de extractos}

La macroalga Sargassum spp. se obtuvo del Caribe Mexicano por el proveedor Baja Kelp, se dejó secar al sol durante $24 \mathrm{~h}$ y se hizo un lavado con abundante agua para eliminar partículas externas, se secó en un horno a $60{ }^{\circ} \mathrm{C}$ y se pulverizó hasta obtener un tamaño de partícula menor a 0.5 $\mathrm{mm}$. Posteriormente, se realizaron 17 extracciones siguiendo la metodología descrita por Ruíz et al. (2015) con algunas modificaciones. En un reactor tipo batch se colocaron $5 \mathrm{~g}$ de muestra seca de SAR con $100 \mathrm{~mL}$ de solvente, se consideraron tres factores de variación: proporción del solvente $(0,25$ y $50 \%$ de etanol), tiempo de extracción (0, 15 y $30 \mathrm{~min})$ y temperatura de extracción (120, 140 y $160{ }^{\circ} \mathrm{C}$ ), los cuales se establecieron mediante un diseño central compuesto (Tabla 1).

Tabla 1. Descripción de los tratamientos utilizados en la prueba en plántulas de tomate.

\begin{tabular}{lccc}
\hline Tratamiento & $\begin{array}{c}\mathrm{T} \\
\left({ }^{\circ} \mathrm{C}\right)\end{array}$ & $\begin{array}{c}\text { TE } \\
(\mathrm{min})\end{array}$ & $\begin{array}{c}\text { Etanol } \\
(\%)\end{array}$ \\
\hline E1 & 120 & 0 & 0 \\
E2 & 120 & 0 & 50 \\
E3 & 120 & 30 & 50 \\
E4 & 120 & 30 & 0 \\
E5 & 160 & 0 & 0 \\
E6 & 160 & 0 & 50 \\
E7 & 160 & 30 & 50 \\
E8 & 160 & 30 & 0 \\
E9 & 140 & 15 & 25 \\
E10 & 140 & 15 & 25 \\
E11 & 140 & 15 & 25 \\
E12 & 120 & 15 & 25 \\
E13 & 160 & 15 & 25 \\
E14 & 140 & 0 & 25 \\
E15 & 140 & 30 & 25 \\
E16 & 140 & 15 & 0 \\
E17 & 140 & 15 & 50 \\
\hline T: Temperatura; & TE: & Tiempo de \\
extracción. & & &
\end{tabular}

\section{Ubicación y establecimiento del experimento}

El estudio se realizó durante el periodo de mayo-octubre del 2020 en un invernadero tipo capilla. Se usaron semillas de $S$. lycopersicum variedad híbrido El CID F1, sembradas en charolas de poliestireno. A los 24 días después de la siembra, se trasplantaron en contenedores de unicel de $0.8 \mathrm{~L}$, usando como sustrato peat moss y perlita (1:1), la nutrición fue provista a través de la solución Steiner (Steiner 1961) de acuerdo a la etapa fenológica. EI experimento tuvo una duración de 43 DDT, siguiendo un diseño completamente al azar con 20 tratamientos y 10 repeticiones por tratamiento. Se evaluaron 17 extractos de SAR (E1-E17, Tabla 1) aplicados en las plántulas infectadas con F. oxysporum, un testigo infectado en plántulas inoculadas con $F$. oxysporum (TI), un producto comercial a base de Ascophyllum nodosum aplicado en plántulas infectadas con $F$. oxysporum (TCl) y un testigo absoluto (T0) en plántulas sin aplicaciones y sin infección. La aplicación de los tratamientos fue a los 7, 12, 22, 33 y 43 DDT asperjándose a punto de goteo en el haz y envés de las hojas a una concentración de $3 \mathrm{Lha}^{-1}$. La inoculación de $F$. oxysporum se realizó a los 14 DDT, utilizando cinco explantes de $0.7 \mathrm{~cm}$ de diámetro por contenedor, los cuales se colocaron a lado de la raíz.

\section{Variables evaluadas}

La inducción de tolerancia de los extractos de SAR se evaluó en términos de incidencia de la enfermedad considerando la presencia de plantas sanas y enfermas, además, se midió la severidad de los síntomas de la enfermedad causada por $F$. oxysporum de acuerdo a la escala propuesta por Diener y Ausubel (2005), donde: $0=$ planta muerta $(100 \%) ; 1=$ hojas viejas muertas y hojas jóvenes con crecimiento detenido $(80 \%) ; 2=$ hojas viejas cloróticas y hojas jóvenes con crecimiento detenido (60\%); $3=$ hojas viejas con clorosis vascular y hojas jóvenes con crecimiento detenido (40\%); 4 = peciolos de hojas con crecimiento detenido (20\%); 5 = sin síntomas visibles $(0 \%)$. También se determinaron variables como diámetro de tallo con un vernier digital $(\mathrm{mm})$, número de hojas y altura de planta con un flexómetro (cm), las cuales se determinaron a los 43 DDT.

\section{Análisis estadístico de datos}

Los datos se analizaron con el programa estadístico InfoStat 2020, para determinar diferencias entre medias se realizó la prueba LSD Fisher a una probabilidad de $\mathrm{p} \leq 0.05$. 


\section{RESULTADOS Y DISCUSIÓN}

\section{Variables de crecimiento de las plántulas Diámetro de tallo}

En la variable de diámetro de tallo se observaron diferencias significativas entre los tratamientos evaluados a los 43 DDT (Figura 1). Los tratamientos TCI, E1, E3, E4, E8, E9, E12, E13 y E17 fueron estadísticamente similares al testigo absoluto (T0). Sin embargo, los tratamientos E2, E5, E6, E7, E10, E11, $\mathrm{E} 14, \mathrm{E} 15$ y E16 fueron estadísticamente similares al testigo infectado ( $\mathrm{TI})$, los cuales redujeron el diámetro de tallo y por lo tanto el vigor de la plántula. Los tratamientos de mayor diámetro fueron el TCI, E12 y E17 con promedio de $4.9 \mathrm{~mm}$. Se tiene poca información que pruebe la capacidad de resistencia frente al estrés biótico de extractos de Sargassum, pero se ha demostrado que los extractos de Sargassum, actúan como bioestimulantes en los procesos fenológicos de las plantas. Al respecto, Uribe-Orozco et al. (2018), demostraron que al ser aplicado en bajas dosis (3$9 \mathrm{~g} \mathrm{maceta}^{-1}$ ) mostró un incremento en el sistema radicular de plantas de cilantro. También se ha demostrado que la aplicación de extractos de algas promueve efectos benéficos en el crecimiento y desarrollo de los cultivos, reflejándose en el vigor de las plantas (Hernández-Herrera et al. 2014).

\section{Número de hojas}

Se observó reducción en el número de hojas en función de la edad de las plantas y el avance de la infección, debido a que los síntomas distintivos ocasionados por $F$. oxysporum son visibles en las hojas inferiores que desarrollan clorosis. La aplicación de algunos extractos promovió un efecto estimulante al permitir un número de hojas igual al testigo absoluto, como en los tratamientos E1, E3, E4, E8, E9, E12, E13, E14, E16, E17 y el TCl a los 43 DDT con un promedio de nueve hojas sanas por plántula (Figura 2). Lo anterior, está relacionado con la concentración de compuestos bioactivos en los extractos de algas, ya que modifican el metabolismo de las plantas, lo que mejora el crecimiento y desarrollo de los cultivos (Hernández-Herrera et al. 2018). Por otro lado, el menor número de hojas por planta a los 43 DDT en los extractos E2 y E11 fue similar al testigo infectado, en el que no hubo resistencia al ataque de $F$. oxysporum. En conjunto con el estrés ocasionado por patógenos, algunos autores asocian la reducción del vigor de los cultivos a la aplicación de extractos, en los cuales las condiciones de procesamiento y el contenido de compuestos en altas concentraciones, produce efectos negativos en las plantas (Sutharsan et al. 2016).

\section{Altura de plantas}

Para la altura de las plántulas a los 43 DDT se encontraron diferencias significativas entre tratamientos (Figura 3), observando un crecimiento más próximo al testigo absoluto en la aplicación de los extractos E12, E13, E17 y TCl con altura promedio de $39 \mathrm{~cm}$ por planta. Los resultados observados son de importancia, ya que las plantas infectadas con el patógeno $(\mathrm{TI})$ presentaron un crecimiento detenido importante, sin embargo, algunos tratamientos (E12, $E 13, E 17)$ evidenciaron un efecto estimulante de los extractos de Sargassum spp. Mientras que las plantas que presentaron la menor altura fueron las del extracto E1. La literatura señala que la combinación de extractos de algas pardas es ideal en la producción de cultivos, como el estudio realizado por Sujatha et al. (2015) quienes reportan la aplicación de un complejo de algas pardas, incluidas Sargassum, con las que obtuvieron una inducción del crecimiento de plantas de chile verde, nabos y piña, bajo condiciones de desarrollo normales.

\section{Incidencia y severidad}

En la evaluación de incidencia y severidad de la enfermedad se encontraron diferencias significativas a los 43 DDT. En la Figura 4 observamos que el E12 mostró una reducción del 17\% de la severidad en comparación con el testigo infectado, lo que indica que las plántulas de este tratamiento tenían peciolos de hojas con crecimiento detenido, siendo el tratamiento E12 el único similar estadísticamente al testigo absoluto. El efecto anterior está relacionado con la presencia de compuestos bioactivos que actúan como bioestimulantes promoviendo la resistencia al estrés biótico (Vera et al. 2011). Al 


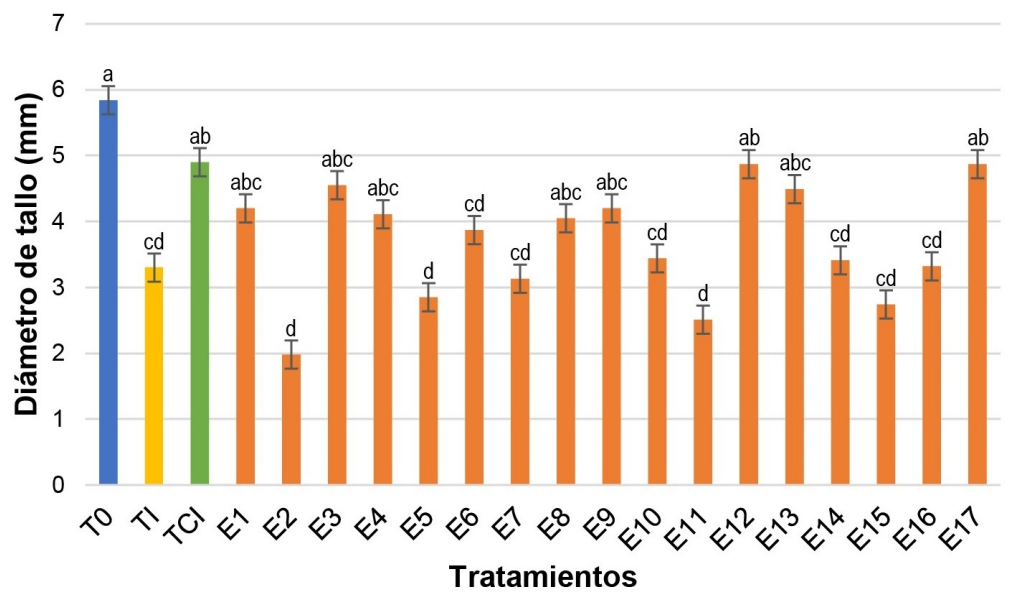

Figura 1. Diámetro de tallo en plántulas de tomate tratadas con aplicaciones foliares de extractos de Sargassum spp. a los 43 DDT. Letras diferentes indican diferencias significativas entre tratamientos (LSD, $p \leq 0.05)$.

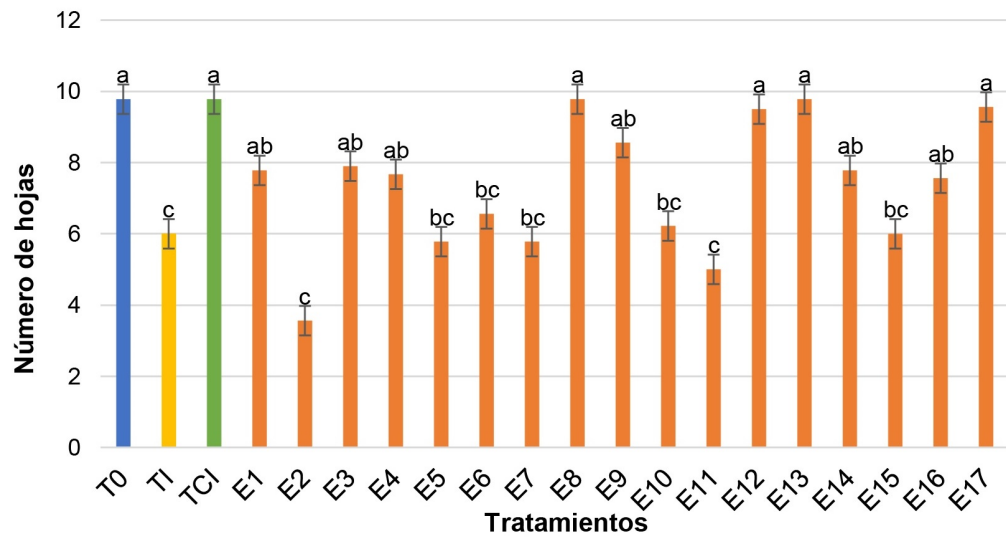

Figura 2. Número de hojas en plántulas de tomate tratadas con aplicaciones foliares de extractos de Sargassum spp. a los 43 DDT. Letras diferentes indican diferencias significativas entre tratamientos (LSD, $p \leq 0.05$ ).

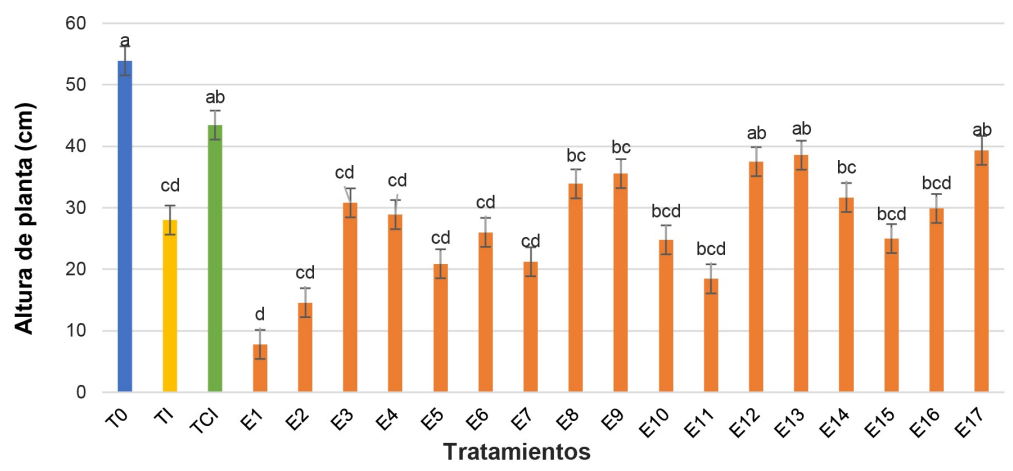

Figura 3. Altura de plántulas de tomate tratadas con aplicaciones foliares de extractos de Sargassum spp. a los 43 DDT. Letras diferentes indican diferencias significativas entre tratamientos (LSD, $\mathrm{p} \leq 0.05)$. 


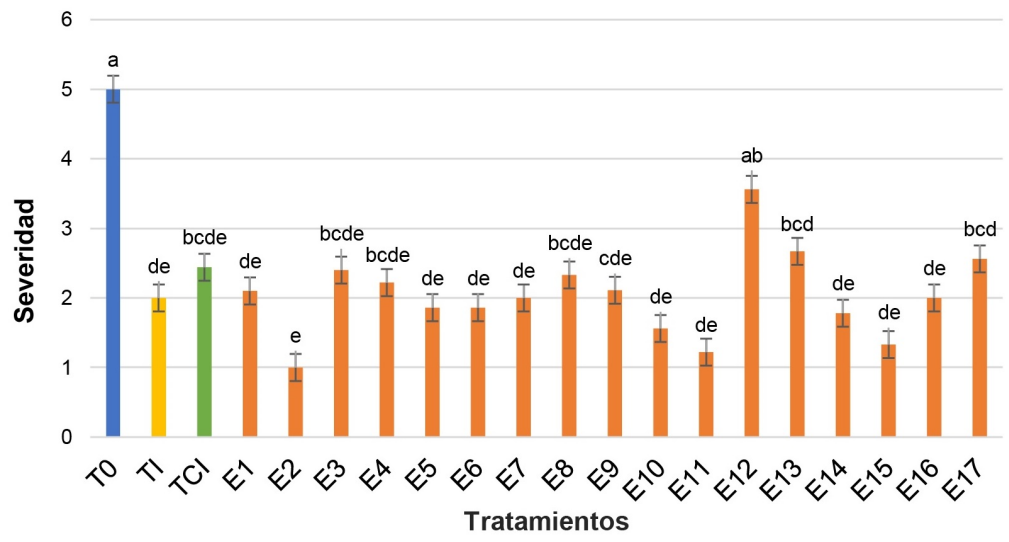

Figura 4. Severidad de F. oxysporum en plántulas de tomate tratadas con aplicaciones foliares de extractos de Sargassum spp. a los 43 DDT. Letras diferentes indican diferencias significativas entre tratamientos (LSD, $p \leq 0.05$ ).

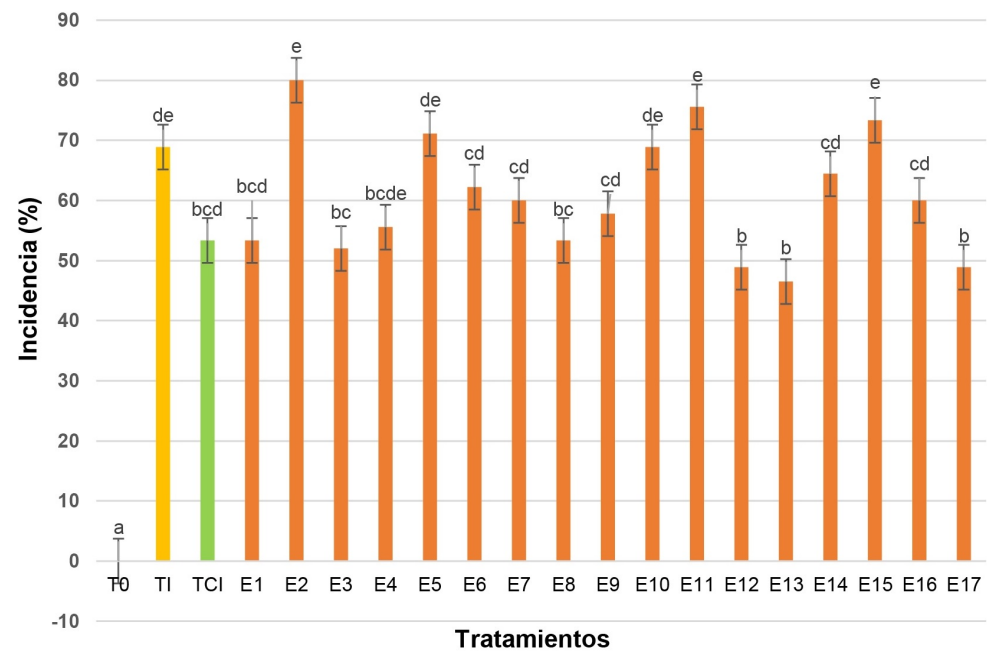

Figura 5. Porcentaje de incidencia de F. oxysporum en plántulas de tomate tratadas con aplicaciones foliares de extractos de Sargassum spp. a los 43 DDT. Letras diferentes indican diferencias significativas entre tratamientos (LSD, $p \leq 0.05$ ).

respecto, Pérez-Madruga et al. (2020) demostraron que aplicaciones foliares de un extracto de Ascophylum nodosum redujeron la infección por Phytophthora capsici en Capsicum y Plasmopara viticola. En cuanto a la incidencia de la enfermedad (Figura 5), se observó un efecto generalizado en los tratamientos infectados al desarrollar la enfermedad, sin embargo, los extractos que presentaron la menor incidencia fueron el E12, E13 y E17 con un promedio de $47 \%$; con lo que se comprueba que con la aplicación de extractos de SAR se promueve la inducción de resistencia a estrés por $F$. oxysporum, además de tener un efecto sobre el crecimiento y desarrollo de la planta. Lo que se debe a que algunas plantas tienen la capacidad de sintetizar y acumular en sus órganos metabolitos secundarios, que intervienen en interacciones complejas entre organismos vivos y que además tienen efectos estimulantes sobre el crecimiento de las plantas (García-Enciso et al. 2018). El extracto que mejores resultados presentó, en base a las variables agronómicas y de incidencia y severidad, fue el E12 (proporción etanol-agua 1:3), el cual fue seleccionado para su caracterización en términos bioquímicos. Los extractos E12, E13, E17 y 
TCl de Sargassum spp. promovieron el crecimiento y desarrollo en plántulas de tomate, con valores similares al testigo absoluto. El extracto E12 mostró la mayor efectividad en la inducción de resistencia a F. oxysporum, por lo que representa una alternativa para la formulación de un bioestimulante de origen biológico que mejore la resistencia al estrés biótico en plántulas de tomate como consecuencia de los compuestos bioactivos y de la actividad sinergica en- tre la matriz de compuestos químicos que lo integran.

\section{AGRADECIMIENTOS}

Al Consejo Nacional de Ciencia y Tecnología (CONACYT) número de beca 1032001. Maestría en Ciencias en Horticultura, Universidad Autónoma Agraria Antonio Narro. Saltillo, México.

\section{LITERATURA CITADA}

Ali Q, Shehzad F, Waseem M, Shahid S, Hussain Al, Haider MZ, Perveen R (2020) Plant-based biostimulants and plant stress responses. In: Hasanuzzaman M (ed.) Plant ecophysiology and adaptation under climate change: Mechanisms and perspectives I. Springer. Singapore. pp: 625-661.

Bouissil S, Alaoui-Talibi E, Pierre G, Michaud P, El Modafar C, Delattre C (2020) Use of alginate extracted from Moroccan brown algae to stimulate natural defense in date palm roots. Molecules 25(3): 720. DOI: 10.3390/molecules25030720.

Campos EV, Proença PL, Oliveira JL, Bakshi M, Abhilash PC, Fraceto LF (2019) Use of botanical insecticides for sustainable agriculture: Future perspectives. Ecological Indicators 105: 483-495.

Diener AC, Ausubel FM (2005) Resistance to Fusarium oxysporum 1, a dominant Arabidopsis disease-resistance gene, is not race specific. Genetics 171: 305-321.

Dmytryk A, Chojnacka K (2018) Algae as fertilizers, biostimulants, and regulators of plant growth. In: Chojnacka K, Wieczorek PP, Schroeder G, Michalak I (eds.) Algae biomass: Characteristics and applications. Springer, Cham. Poland. pp: 115-122.

García Enciso EL, Robledo Olivo A, Benavides Mendoza A, Solís Gaona S, González Morales S (2018) Efecto de elicitores de origen natural sobre plantas de tomate sometidas a estrés biótico Revista Mexicana de Ciencias Agrícolas Vol. Esp. 20: 4212-4221.

González de Molina M, Soto Fernandez D, Infante-Amate J, Aguilera E, Vila Traver J, Guzmán GI (2017) Decoupling food from land: the evolution of Spanish agriculture from 1960 to 2010. Sustainability 9: 2348. DOI: $10.3390 /$ su9122348.

Hernández-Herrera RM, Santacruz-Ruvalcaba F, Briceño-Domínguez DR, Di Filippo-Herrera DA, HernándezCarmona G (2018) Seaweed as potential plant growth stimulants for agriculture in Mexico. Hidrobiologica 28: $129-140$.

Hernández-Herrera RM, Virgen-Calleros G, Ruiz-López M, Zañudo-Hernández J, Délano-Frier JP, SánchezHernández C (2014) Extracts from green and brown seaweeds protect tomato (Solanum lycopersicum) against the necrotrophic fungus Alternaria solani. Journal of Applied Phycology 26: 1607-1614.

Juárez-Maldonado A, Ortega-Ortíz H, Morales-Díaz AB, González-Morales S, Morelos-Moreno Á, SandovalRangel A, Benavides-Mendoza A (2019) Nanoparticles and nanomaterials as plant biostimulants. International Journal of Molecular Sciences 20: 162. DOI: 10.3390/ijms20010162. 
Kumar S, Sachdeva S, Bhat KV, Vats S (2018) Plant responses to drought stress: Physiological, biochemical and molecular basis. In: Aroca R (ed.) Biotic and abiotic stress tolerance in plants. Springer. Singapore. pp: $1-4$.

Maluin FN, Hussein MZ (2020) Chitosan-based agronanochemicals as a sustainable alternative in crop protection. Molecules 25: 1611. DOI: 10.3390/molecules25071611.

Melo PC, Collela CF, Sousa T, Pacheco D, Cotas J, Gonçalves AMM (2020) Seaweed-based products and mushroom $\beta$-glucan as tomato plant immunological inducers. Vaccines 8: 524. DOI: 10.3390/vaccines8030524.

Pérez-Madruga Y, López-Padrón I, Reyes-Guerrero Y (2020) Las Algas como alternativa natural para la producción de diferentes cultivos. Cultivos Tropicales 41: 1-20.

Rehman A, Korejo F, Sultana V, Ara, J, Ehteshamul-Haque S (2017) Induction of systemic resistance in cotton by the plant growth promoting rhizobacteria (PGPR) and seaweed against charcoal rot disease. Pakistan Journal of Botany 49: 347-353.

Ammar N, Nefzi A, Jabnoun-Khiareddine H, Daami-Remadi M (2017) Control of fusarium dry rot incited by Fusarium oxysporum f. sp. tuberosi using Sargassum vulgare aqueous and organic extracts. Journal of Microbial \& Biochemical Technology 9: 200-208.

Rodríguez-Martínez RE, van Tussenbroek B, Jordán-Dahlgren E (2016) Afluencia masiva de sargazo pelágico a la costa del Caribe mexicano (2014-2015). En: Ernesto García-Mendoza, Quijano-Scheggia SI, OlivosOrtiz A, Núñez-Vázquez EJ (eds.) Florecimientos algales nocivos en México. CICESE. Ensenada, México. pp: 352-365.

Ruiz HA, Rodríguez-Jasso RM, Aguedo M, Kadar Z (2015) Hydrothermal pretreatments of macroalgal biomass for biorefineries. In: Prokop A, Bajpai EK, Zappi ME (eds.) Algal biorefineries, Springer Cham. USA. pp: 467-491.

Singh VK, Singh AK, Kumar A (2017) Disease management of tomato through PGPB: current trends and future perspective. 3 Biotech 7: 255. DOI: 10.1007/s13205-017-0896-1.

Steiner AA (1961) A universal method for preparing nutrient solutions of a certain. Plant and Soil 15: 134-154.

Sujatha K, Vijayalakshmai V, Suganthi A (2015) Comparative efficacy of brown, red and green seaweed extracts on low vigour sunflower (Helianthus annus L.) var. TN (SUF) 7 seeds. African Journal of Agricultural Research 10: 2165-2169.

Summerell BA (2019) Resolving Fusarium: current status of the genus. Annual Review of Phytopathology 57: 323-339.

Sutharsan S, Nishanthi S, Srikrishnah S (2016) Effect of seaweed extract (Sargassum crassifolium) foliar application on the performance of Lycopersicon esculentum Mill. in sandy regosol of Batticaloa. American-Eurasian Journal of Agriculatural \& Environmental Sciences 14: 1386-1396.

Uribe-Orozco ME, Mateo-Cid LE, Mendoza-González AC, Amora-Lazcano EF, González-Mendoza D, DuránHernández D (2018) Efecto del alga marina Sargassum vulgare C. Agardh en suelo y el desarrollo de plantas de cilantro. Idesia 36: 69-76.

Vera J, Castro J, Gonzalez A, Moenne A (2011) Seaweed polysaccharides and derived oligosaccharides stimulate defense responses and protection against pathogens in plants. Marine Drugs 9: 2514-2525. 